\title{
Increasing implementation and delivery of pulmonary rehabilitation: key messages from the new ATS/ERS policy statement
}

\author{
Ioannis Vogiatzis ${ }^{1}$, Carolyn L. Rochester ${ }^{2,3}$, Martijn A. Spruit ${ }^{4,5}$, \\ Thierry Troosters ${ }^{6}$ and Enrico M. Clini ${ }^{7}$ on behalf of the American Thoracic \\ Society/European Respiratory Society Task Force on Policy in Pulmonary \\ Rehabilitation $^{8}$
}

\begin{abstract}
Affiliations: ${ }^{1}$ National and Kapodistrian University of Athens, Department of Physical Education and Sports Science; 1st Department of Respiratory Medicine, Pulmonary Rehabilitation Unit; 1st Department of Critical Care Medicine, Pulmonary Rehabilitation Centre, Athens, Greece. ${ }^{2}$ Yale University School of Medicine, New Haven, CT, USA. ${ }^{3}$ VA Connecticut Healthcare System, West Haven, CT, USA. ${ }^{4}$ Dept of Research and Education, $\mathrm{CIRO}+$, Center of Expertise for Chronic Organ Failure, Horn, The Netherlands. ${ }^{5}$ REVAL-Rehabilitation Research Center, BIOMED - Biomedical Research Institute, Faculty of Medicine and Life Sciences, Hasselt University, Diepenbeek, Belgium. ${ }^{6}$ Katholieke Universiteit Leuven, Department of Rehabilitation Sciences and University Hospital Leuven, Leuven, Belgium. ${ }^{7}$ Dept. of Medical and Surgical Sciences, University of Modena and Reggio Emilia, Modena, Italy. ${ }^{8}$ A full list of members of the ATS/ERS Task Force on Policy in Pulmonary Rehabilitation can be found in the Acknowledgements section.
\end{abstract}

Correspondence: loannis Vogiatzis, University of Athens, 1st Department of Critical Care Medicine, 3 Ploutarhou Str. 10675, Athens, Greece. E-mail: gianvogaphed.uoa.gr

@ERSpublications

The goal of the New Policy Statement is to expand provision of pulmonary rehabilitation to suitable patients worldwide http://ow.ly/ZcBJv

In December 2015, the Official American Thoracic Society (ATS)/European Respiratory Society (ERS) Policy Statement on Enhancing Implementation, Use and Delivery of Pulmonary Rehabilitation was published [1] with the aim of providing policy recommendations to increase implementation and delivery of pulmonary rehabilitation worldwide. Major areas addressed included increasing healthcare professional, payer and patient awareness and knowledge of pulmonary rehabilitation, increasing patient access to pulmonary rehabilitation, improving quality of pulmonary rehabilitation programmes and future research directions to advance evidence-based policy in pulmonary rehabilitation. This ATS/ERS document was developed via an iterative consensus process by an ad hoc Task Force on Policy in pulmonary rehabilitation comprised of experts from the ATS Pulmonary Rehabilitation Assembly, the ERS Rehabilitation and Chronic Care Group, the ATS and ERS Documents Development and Implementation Committees, representatives from the European Lung Foundation (ELF) and primary care representatives from the USA and Europe between May 2013 and January 2015. Input was obtained via informal surveys from patients, patient advocacy groups, (including the ATS Public Advisory Roundtable and ELF), insurance payers, as well as primary and pulmonary specialty healthcare providers. The Policy Statement was approved by the Board of Directors of the ATS and the Science Council and Executive Committee of the ERS. This editorial provides European Respiratory Journal readers with a concise reflection on the key issues addressed and summarises the policy recommendations made in the ATS/ERS Policy Statement [1] to enhance implementation, use and delivery of pulmonary rehabilitation.

Received: Dec 202015 | Accepted after revision: Feb 172016

Conflict of interest: None declared.

Support statement: funding was provided by ERS (TF-2014-11). Funding information for this article has been deposited with FundRef.

Copyright OERS 2016 
Increasing awareness and knowledge of pulmonary rehabilitation

Suboptimal healthcare professional awareness and knowledge of pulmonary rehabilitation is a major barrier to patient referral [2]. In a majority of countries worldwide, training in pulmonary rehabilitation is not mandatory or standardised, and exposure to pulmonary rehabilitation is limited. In turn, healthcare professionals in clinical practice are often not familiar with the science, process and benefits of pulmonary rehabilitation, and as such do not recommend it for suitable patients. There is a clear need for more standardised, formal training in pulmonary rehabilitation for healthcare professionals. Training requirements should be specified in national training curriculum documents, and educational authorities from respiratory, primary care, nursing and other allied healthcare professionals' societies should collaborate to develop specific curricula for pulmonary rehabilitation training for their respective healthcare trainees. Consistency of training should be maintained in programmes for each healthcare discipline and across disciplines. More training opportunities in pulmonary rehabilitation (consistent with evidence-based guidelines) are also needed for healthcare professionals already in clinical practice.

Although pulmonary rehabilitation is one of the most cost-effective therapies for individuals with chronic respiratory disease (figure 1) [3], suboptimal payer knowledge and awareness of pulmonary rehabilitation [4] contributes to a large shortfall in funding for pulmonary rehabilitation programmes. Insufficient funding leads to an inadequate number of pulmonary rehabilitation programmes to meet patient needs as well as inadequate resources (e.g. staffing, infrastructure, equipment, space) within existing programmes. Adequate funding is essential for pulmonary rehabilitation programme availability, capacity, effectiveness and viability. Documentation, transparency and communication by healthcare professionals regarding the processes, costs, outcomes and cost-effectiveness of pulmonary rehabilitation to payers are key steps necessary for securing increased funding for pulmonary rehabilitation programmes. Increased dialogue is needed between pulmonary rehabilitation experts, professional societies (such as ERS, ATS and others), payers and health policy authorities regarding the benefits of pulmonary rehabilitation for patients, the cost-effectiveness of pulmonary rehabilitation and the priorities and concerns of payers. More study is also needed regarding the cost-effectiveness of pulmonary rehabilitation relative to other therapies for respiratory disorders other than chronic obstructive pulmonary disease (COPD).

Patients with chronic respiratory diseases who can benefit from PR must be made aware of pulmonary rehabilitation so they can advocate for access to it. Many patients have never heard of pulmonary rehabilitation at all. For many others with disabling dyspnoea and significant functional limitations, the idea of participating in a programme that involves exercise training is counterintuitive and/or frightening. Overall, uptake of and participation in pulmonary rehabilitation by suitable patients remains poor [5-8]. Patients need knowledge about the health benefits of pulmonary rehabilitation and perhaps more importantly, knowledge about the ways by which pulmonary rehabilitation affords these benefits, so as to make informed decisions regarding participation. Language-, education- level- and culturally appropriate education materials for patients prepared in multiple formats (written, internet-based, audio, etc.) regarding the processes and benefits of pulmonary rehabilitation are needed. Family members, other caregivers, friends, coworkers and others can encourage patients to participate in pulmonary rehabilitation if they are aware of it. Broad-scale communication campaigns are also needed to foster public awareness of pulmonary rehabilitation.

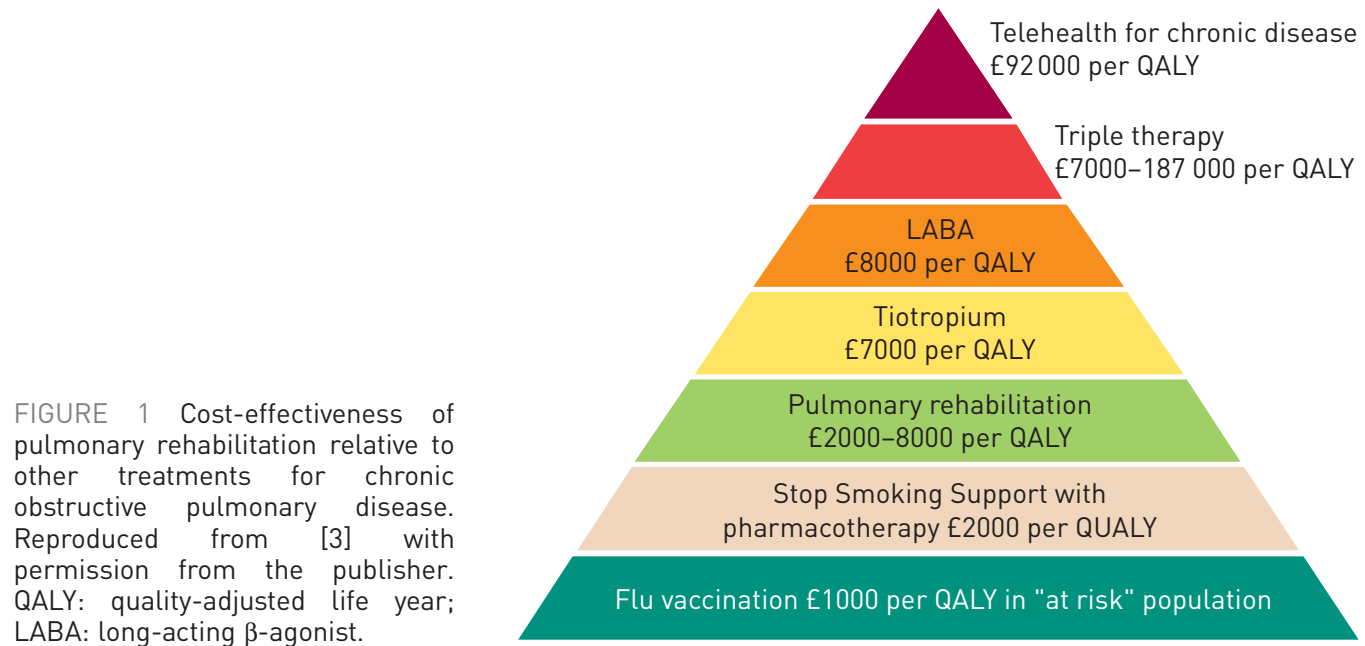




\section{Increasing patient access to pulmonary rehabilitation}

Insufficient access to pulmonary rehabilitation is an important barrier to pulmonary rehabilitation delivery [1]. Access can be limited in several ways. Suitable patients may not be able to enter a programme due to limited programme capacity or may be placed on a long waiting list. Access can also be impaired by pulmonary rehabilitation programmes' geographical location yielding a travel distance that is not acceptable to, affordable by or possible for the patient $[5,6,9]$. This is particularly the case for the frail patient who lacks social support. In addition, local logistics and infrastructure are frequently suboptimal. For example, parking space around programmes is often limited and walking distance to programmes may be inappropriate for patients with advanced chronic respiratory diseases. Attention to these issues and identification of novel ways of providing multidisciplinary rehabilitation (e.g., in the community or potentially through tele-rehabilitation) [1] may potentially provide greater capacity of pulmonary rehabilitation, although more evidence proving the efficacy of novel ways to deliver pulmonary rehabilitation is needed.

Also, to be successful, healthcare payers will need to support these innovative forms of rehabilitation. Payers may be relatively slow in adopting new concepts. For example, reimbursement for pulmonary rehabilitation is often reserved for patients with COPD suffering from severe airflow obstruction, yet presence or severity of airflow obstruction alone should not be a required selection criterion for pulmonary rehabilitation $[1,10]$. Based on the scientific evidence, patients with respiratory disorders who suffer from symptoms despite optimal pharmacotherapy, inactivity, skeletal muscle dysfunction [11] or other non-respiratory consequences of their disease should have access to pulmonary rehabilitation [10, 12]. This includes, but is not limited to, symptomatic patients with COPD and those with non-COPD respiratory disorders such as interstitial lung disease, pulmonary hypertension, cystic fibrosis, or lung cancer $[10,12]$. Furthermore, pulmonary rehabilitation should be made accessible to any patient with COPD who has been hospitalised with an acute exacerbation $[1,10]$.

Several steps are needed at multiple levels to increase access to pulmonary rehabilitation. At the local level, existing pulmonary rehabilitation programmes need to be made as accessible as possible; more and well-equipped programmes (in terms of resources, logistics, availability and expertise of healthcare providers) are needed to accommodate more patients. At the regional level, more programmes need to be established in the community of the patient where programmes are lacking. In addition, novel techniques need to be validated that can bring programmes to patients whenever needed (innovative level) and these programmes need to be endorsed with funding that allows establishment of sustainable quality programmes (reimbursement level). Increased healthcare payer acceptance of a broader range of suitable candidates for participation in pulmonary rehabilitation is also needed.

\section{Ensuring quality of pulmonary rehabilitation programs}

To qualify as pulmonary rehabilitation, programmes must include, at the very least, comprehensive baseline and post-pulmonary rehabilitation outcome assessments; a structured and supervised exercise training programme; an education/behavioral programme intended to foster long-term health-enhancing behaviour; and provision of recommendations for home-based exercise and physical activity [4, 10, 13-21]. Moreover, the pulmonary rehabilitation team must have demonstrated skills in provision of pulmonary rehabilitation [22]. Interestingly, large heterogeneity exists in content and organisational aspects of pulmonary rehabilitation programmes around the world [5]. Pulmonary rehabilitation programme certification is a means of ensuring that pre-determined, international standards are met. Certification would also enable emphasis on and dissemination of quality standards for pulmonary rehabilitation programmes to healthcare facilities, healthcare professionals, payers and policy makers.

The quality of pulmonary rehabilitation programmes is demonstrated by their success in improving patient outcomes. Therefore, each pulmonary rehabilitation programme needs to start with a comprehensive patient assessment to allow delivery of individualised rehabilitation according to each patient's needs. To assess programme quality, clinical outcomes must also be measured for individual patients on completion of pulmonary rehabilitation $[23,24]$. At a minimum, patients' functional exercise capacity, dyspnoea and health status should be assessed $[10,13,16,19,25,26]$. The selection and standardisation of outcome measures for the purpose of programme comparison and benchmarking will vary among different jurisdictions, but should be guided by the respiratory scientific community based on disease-relevant published evidence.

Evidence of safety should be assessed based on standard operating policies, risk assessments and critical incident reporting. Pulmonary rehabilitation programme directors should conduct regular internal audits of programme process and outcomes. Pulmonary rehabilitation programmes should also collect and respond to information on patient experience, needs and satisfaction prior to, during and after pulmonary rehabilitation.

Accordingly, pulmonary rehabilitation programmes should follow relevant evidence-based clinical guidelines and demonstrate the measurement of standard outcomes to document benefits, quality and 
safety. Core sets of key process and performance indicators need to be established to enable (international) bench marking, programme accreditation, and to justify reimbursement from payers.

\section{Future research directions}

Key areas for future research have been identified that may influence commissioning policy for pulmonary rehabilitation and thus may potentially increase delivery and uptake of pulmonary rehabilitation by eligible patients [1]. Priority should be given to research related to cost effectiveness [3] and long-term health outcomes associated with delivery of pulmonary rehabilitation for people with early stages of respiratory disease, provision of pulmonary rehabilitation for respiratory disorders other than COPD [10, 12], optimal duration and frequency of repeated pulmonary rehabilitation courses over time, as well as the identification of those patients who are expected to benefit most from participation in pulmonary rehabilitation [15-18].

In regions or healthcare systems where traditional models of pulmonary rehabilitation are not feasible, research should focus on the effectiveness of alternative models of pulmonary rehabilitation such as tele-rehabilitation or home-based rehabilitation supported by pulmonary rehabilitation programme staff with or without specialised exercise equipment for exercise training. A cost analysis of these alternative models should consider exacerbation and hospitalisation rates as well as healthcare resource utilisation.

Future research should also address strategies for optimising the benefits of pulmonary rehabilitation for patients with severe psychological and multiple medical comorbidities. In addition, research should tackle the barriers and facilitators of pulmonary rehabilitation programme referrals, accessibility, enrolment and adherence for patients.

Priorities for pulmonary rehabilitation-related research should be articulated and documented by pulmonary rehabilitation experts actively involved in ERS and ATS [1, 10], other national/continental professional organisations. These priorities should then be communicated to patient advocacy groups and funding agencies via stakeholder forums and workshops. Healthcare professional experts in pulmonary rehabilitation should also lobby professional societies, advocacy organisations and federal agencies to increase funding for pulmonary rehabilitation research.

\section{Pulmonary rehabilitation across Europe: present and future}

A recent international survey study involving over 400 centres in 40 countries, has addressed differences in content and organisation of pulmonary rehabilitation programmes worldwide [5]. Currently, in particular, the sources of funding and reimbursement, composition of pulmonary rehabilitation staff, and content delivered within rehabilitation programmes for similar patients appears different between Europe and the Americas [5].

In Europe, hospital-based inpatient programmes with a high allocation of multidisciplinary staff are a more common option as a setting to deliver pulmonary rehabilitation compared with the USA, where free-standing outpatient or community-based pulmonary rehabilitation programmes are more commonly in use. In European countries, COPD is the predominant diagnosis for which patients are referred to pulmonary rehabilitation, whereas individuals with other respiratory conditions such as lung fibrosis, pulmonary hypertension or those recovering from lung-resection are less commonly enrolled in the standard programmes.

Factors that likely contribute to differences in pulmonary rehabilitation structure, content, and resource allocation relate to the local and national healthcare policy, a generally nihilistic public view regarding care for advanced chronic respiratory diseases [4], and a lack of homogeneity in training of healthcare professionals. It is therefore recommended that national respiratory societies in Europe should coordinate the development of a common advocacy strategy to facilitate incorporation of outpatient pulmonary rehabilitation into the respective healthcare systems as a "standard of care" component of the integrated care of patients with chronic lung diseases [1]. Educational authorities from respiratory, primary care, nursing and other allied healthcare professionals' societies need to collaborate in developing specific curricula for pulmonary rehabilitation training for their respective healthcare trainees [1]. Furthermore, these professionals' societies in different continents should establish national training programme curricular registries to coordinate and monitor progress toward curricula development to harmonise the different national pulmonary rehabilitation practices.

In Europe (and elsewhere) there is an insufficient number of physiotherapists and other healthcare professionals specifically involved in pulmonary rehabilitation who are qualified to deliver pulmonary rehabilitation to respiratory patients. The non-standardised academic training of these healthcare professionals is a crucial aspect of the problem [27]. Important educational projects are ongoing across Europe and North America in effort to fill this gap [28, 29]. 
The current evidence stresses the importance of the future development of process and performance metrics to monitor pulmonary rehabilitation programmes, to be able to start international benchmarking, and to provide recommendations for international standards based on evidence and best practice. This will certainly help to promote an internationally shared standard for education, organisation and resource allocation in the field of pulmonary rehabilitation.

\section{Conclusions}

The physiological, symptom-reducing, psychosocial and health-economic benefits of pulmonary rehabilitation have been demonstrated convincingly across multiple outcome areas for patients with chronic respiratory diseases $[10,12,13,16,17,19,26,30]$. The scientific rationale for and basis underlying these benefits has been well documented [10, 11]. Yet, many, if not most suitable patients lack access to pulmonary rehabilitation programmes, are not referred to pulmonary rehabilitation by healthcare professionals, and/or are not aware of how or the extent to which pulmonary rehabilitation may improve their symptoms, functional status and quality of life. As highlighted in the new Official ATS/ERS Policy Statement: Enhancing Implementation, Use and Delivery of Pulmonary Rehabilitation [1], and as discussed above, many factors contribute to the gap between the science and benefits of pulmonary rehabilitation and the actual delivery of pulmonary rehabilitation services. Disturbingly, variable and typically insufficient access to pulmonary rehabilitation creates unacceptable disparities in quality of evidence-based healthcare. Therefore, a change in the status-quo regarding pulmonary rehabilitation is necessary. The goal of the ATS/ ERS Task force on Policy in Pulmonary Rehabilitation is to expand provision of pulmonary rehabilitation to suitable individuals worldwide. The new ATS/ERS Policy Statement [1] identifies key factors contributing to the existing worldwide shortfall in pulmonary rehabilitation and provides recommendations and actionable items regarding processes central to enhancing its availability and delivery to patients. The way forward is not simple and will take time. An increase in funding and resources for pulmonary rehabilitation is essential. Collaborative efforts of healthcare professionals across multiple disciplines, professional societies (e.g. respiratory, primary care, educational, allied health professional and others), payers, patients, patient advocacy groups and the general public are needed. Increased provision of pulmonary rehabilitation as an evidence-based, standard component of the overall integrated care of symptomatic patients with chronic respiratory diseases worldwide will not only improve the physical and emotional health and quality of life of individual participants, but will also markedly improve the quality of patient care across the trajectory of illness and over time has the potential to significantly reduce healthcare costs.

\section{Acknowledgements}

The ATS/ERS Task Force on Policy in Pulmonary Rehabilitation consisted of: Carolyn L. Rochester: Yale University School of Medicine, New Haven, CT, USA, and VA Connecticut Healthcare System, West Haven, CT, USA; Ioannis Vogiatzis: National and Kapodistrian University of Athens, Athens, Greece; Anne E. Holland: La Trobe University Alfred Health and Institute for Breathing and Sleep, Melbourne, Australia; Suzanne C. Lareau: College of Nursing, University of Colorado, Denver, CO, USA; Darcy D. Marciniuk: University of Saskatchewan, Saskatoon, SK, Canada; Milo A. Puhan: Institute of Social and Preventive Medicine, University of Zurich, Zurich, Switzerland; Martijn A. Spruit: CIRO, Horn, the Netherlands; Sarah Masefield: European Lung Foundation, Sheffield, UK; Richard Casaburi: Rehabilitation Clinical Trials Center, Los Angeles Biomedical Research Institute at Harbor, UCLA Medical Center, Torrance, CA, USA; Enrico M. Clini: University of Modena, Modena, Italy; Rebecca Crouch: Duke University Medical Center, Durham, NC, USA; Judith Garcia-Aymerich: Centre for Research in Enviromental Epidemiology (CREAL), Barcelona, Hospital del Mar Research Institute (IMIM), Barcelona, Spain, Universitat Pompeu Fabra, Barcelona, Spain, and CIBER de Epidemiology y Salud Publica (CIBERESP), Spain; Chris Garvey: Seton Medical Center, Daly City, CA, USA; Roger S. Goldstein: University of Toronto, Toronto, ON, Canada; Kylie Hill: School of Physiotherapy and Curtin Health Innovation Research Institute, Curtin University, Lung Institute of Western Australia and Centre for Asthma, Allergy and Respiratory Research, University of Western Australia, Perth, Australia; Michael Morgan: Institute for Lung Health, Glenfield Hospital, University Hospitals of Leicester NHS Trust, Leicester, UK; Linda Nici: Providence VA Hospital, Providence, RI, USA; Fabio Pitta: Universidade Estadual de Londrina, Londina, Brazil; Andrew L. Ries: University of California, San Diego, CA, USA; Sally J. Singh: University of Leicester, Leicester, UK; Thierry Troosters: Katholieke Universiteit Leuven, University Hospital Leuven, Leuven, Belgium; Peter J. Wijkstra: University of Gronigen, Gronigen, the Netherlands; Barbara P. Yawn: Department of Family and Community Health University of Minnesota, Rochester, MN, USA; and Richard L. ZuWallack: St Francis Hospital, Hartford, CT, USA.

\section{References}

1 Rochester CL, Vogiatzis I, Holland AE, et al. An Official American Thoracic Society/European Respiratory Society Policy Statement: Enhancing Implementation, Use, and Delivery of Pulmonary Rehabilitation. Am J Respir Crit Care Med 2015; 192: 1373-1386.

2 Johnston KN, Young M, Grimmer KA, et al. Barriers to, and facilitators for, referral to pulmonary rehabilitation in COPD patients from the perspective of Australian general practitioners: a qualitative study. Prim care Respir J 2013; 22: 319-324.

3 Zoumot Z, Jordan S, Hopkinson NS. Emphysema: time to say farewell to therapeutic nihilism. Thorax 2014; 69: 973-975. 

option=com_content\&view=article\&id=38:pulmonary-rehabilitation\&catid=17:service-delivery\&Itemid=32 Date last updated: December 2016. Date last accessed: February 10, 2016.

5 Spruit MA, Pitta F, Garvey C, et al. Differences in content and organizational aspects of pulmonary rehabilitation programs. Eur Respir J 2013; 43: 1326-1337.

6 Keating A, Lee A, Holland AE. What prevents people with chronic obstructive pulmonary disease from attending pulmonary rehabilitation? A systematic review. Chron Respir Dis 2011; 8: 89-99.

7 Hayton C, Clark A, Olive S, et al. Barriers to pulmonary rehabilitation: characteristics that predict patient attendance and adherence. Respir Med 2013; 107: 401-407.

8 Garvey C, Fullwood MD, Rigler J. Pulmonary rehabilitation exercise prescription in chronic obstructive lung disease: US survey and review of guidelines and clinical practices. J Cardiopulm Rehabil Prev 2013; 33: 314-322.

9 Rochester CL, Spanevello A. Heterogeneity of pulmonary rehabilitation: like apples and oranges - both healthy fruit. Eur Respir J 2014; 43: 1223-1226.

10 Spruit MA, Singh SJ, Garvey C, et al. An official American Thoracic Society/European Respiratory Society statement: key concepts and advances in pulmonary rehabilitation. Am J Respir Crit Care Med 2013; 188: e13-e64.

11 Maltais F, Decramer M, Casaburi R, et al. An official American Thoracic Society/European Respiratory Society statement: update on limb muscle dysfunction in chronic obstructive pulmonary disease. Am J Respir Crit Care Med 2014; 189: e15-e62.

12 Rochester C, Fairburn C, Crouch R. Pulmonary rehabilitation for disorders other than COPD. Clin Chest Med 2014; 35: 369-389.

13 Singh SJ, ZuWallack RL, Garvey C, et al. Learn from the past and create the future: the 2013 ATS/ERS statement on pulmonary rehabilitation. Eur Respir J 2013; 42: 1169-1174.

14 Australian Lung Foundation Pulmonary Rehabilitation Toolkit. www.pulmonaryrehab.com.au/welcome.asp Date last updated: 2009. Date last accessed: February 10, 2016.

15 Jenkins S, Hill K, Cecins NM. State of the art: how to set up a pulmonary rehabilitation program. Respirology 2010; 15: 1157-1173.

16 Bolton CE, Bevan-Smith EF, Blakey JD, et al. British Thoracic Society guideline on pulmonary rehabilitation in adults. Thorax 2013; 68: Suppl. 2, iil-ii30.

17 Marciniuk DD, Brooks D, Butcher S, et al. Optimizing pulmonary rehabilitation in chronic obstructive pulmonary disease: practical issues: a Canadian Thoracic Society Clinical Practice Guideline. Can Respir J 2010; 17: 159-168.

18 Watz H, Pitta F, Rochester CL, et al. An official European Respiratory Society statement on physical activity in COPD. Eur Respir J 2014; 44: 1521-1537.

19 Ries AL, Bauldoff GS, Carlin BW, et al. Pulmonary Rehabilitation: Joint ACCP/AACVPR Evidence-Based Clinical Practice Guidelines. Chest 2007; 131: Suppl. 5, 4S-42S.

20 Garber CE, Blissmer B, Deschenes MR, et al. American College of Sports Medicine position stand. Quantity and quality of exercise for developing and maintaining cardiorespiratory, musculoskeletal, and neuromotor fitness in apparently healthy adults: guidance for prescribing exercise. Med Sci Sports Exerc 2011; 43: 1334-1359.

21 ACSM's. ACSM's guidelines for exercise testing and prescription. Philadelphia, Lippincott Williams \& Wilkins, 2013.

22 Collins EG, Bauldoff G, Carlin B, et al. Clinical competency guidelines for pulmonary rehabilitation professionals: position statement of the American Association of Cardiovascular and Pulmonary Rehabilitation. J Cardiopulm Rehabil Prev 2014; 34: 291-302.

23 Vanfleteren LE, Spruit MA, Groenen M, et al. Clusters of comorbidities based on validated objective measurements and systemic inflammation in patients with chronic obstructive pulmonary disease. Am J Respir Crit Care Med 2013; 187: 728-735.

24 Spruit MA. Augustin IM, Vanfleteren LE, et al. Differential response to pulmonary rehabilitation in COPD: a multidimensional profiling. Eur Respir J 2015; 46: 1625-1635.

25 Singh SJ, Puhan MA, Andrianopoulos V, et al. An official systematic review of the European Respiratory Society/ American Thoracic Society: measurement properties of field walking tests in chronic respiratory disease. Eur Respir $J$ 2014; 44: 1447-1478.

26 Marciniuk DD, Goodridge D, Hernandez P, et al. Managing dyspnea in patients with advanced chronic obstructive pulmonary disease: a Canadian Thoracic Society clinical practice guideline. Can Respir J 2011; 18: 69-78.

27 Balbi B, Ambrosino N, Lazzeri M, et al. Pulmonary rehabilitation in Italy: professional barriers to overcome Eur Respir J 2014; 44: 1382-1383.

28 Pitta F, Mitchell S, Chatwin M, et al. A core syllabus for post-graduate training in respiratory physiotherapy. Breathe 2014; 10: 220-228.

29 Camp PG, Hernandez P, Bourbeau J, et al. Pulmonary rehabilitation in Canada: A report from the Canadian Thoracic Society COPD Clinical Assembly. Can Respir J 2015; 22: 147-152.

30 Lacasse Y, Cates CJ, McCarthy B, et al. This Cochrane Review is closed: deciding what constitutes enough research and where next for pulmonary rehabilitation in COPD. Cochrane Database Syst Rev 2015; 11: ED000107. 